\title{
Imaging of immunogold labeling in cells and tissues by helium ion microscopy
}

\author{
CHIKARA SATO $^{1}$, MARI SATO ${ }^{1}$ and SHINICHI OGAWA ${ }^{2}$ \\ ${ }^{1}$ Biomedical Research Institute, National Institute of Advanced Industrial Science and Technology (AIST), \\ Tsukuba, Ibaraki 305-8566; ${ }^{2}$ Nanoelectronics Research Institute, \\ National Institute of Advanced Industrial Science and Technology (AIST), \\ Tsukuba, Ibaraki 305-8569, Japan
}

Received July 11, 2017; Accepted March 16, 2018

DOI: $10.3892 /$ ijmm.2018.3604

\begin{abstract}
Helium ion microscopy (HIM) scans samples with a fine ion beam exploiting the very short de Broglie wavelength of helium ions. Because the radiation induces only a small sample region to emit secondary electrons (SEs), very high resolution is expected. In order to explore the applications of SE-HIM in biology, COS7 kidney fibroblast cells and C2C12 myoblast cells cultured on a silicon $(\mathrm{Si})$ nitride $(\mathrm{SiN}) /$ Si bilayer were dried and directly observed in high vacuum, without coating or staining. High contrast, high depth-of-field images were obtained revealing the nucleus, endoplasmic reticulum, cytoskeleton and putative mitochondria above a bright background from the support. Gold-tagged antibodies were employed to aid organelle identification. Signals from the gold tags were most clearly distinguishable by secondary electron (SE)-HIM when cells were grown on thin SiN film, and the minimum gap measured between gold particles showed the resolution to be $2 \mathrm{~nm}$. Wheat germ agglutinin-gold labeling revealed clusters of gold particles $\sim 50-200 \mathrm{~nm}$ in diameter on COS7 cells, which might represent assemblies of glycosylated
\end{abstract}

Correspondence to: Dr Chikara Sato, Biomedical Research Institute, National Institute of Advanced Industrial Science and Technology (AIST), 1-1-1 Higashi, Tsukuba, Ibaraki 305-8566, Japan E-mail: ti-sato@aist.go.jp

Dr Shinichi Ogawa, Nanoelectronics Research Institute, National Institute of Advanced Industrial Science and Technology (AIST), 16-1 Onogawa, Tsukuba, Ibaraki 305-8569, Japan

E-mail: ogawa.shinichi@aist.go.jp

Abbreviations: ASEM, atmospheric SEM; BEI, backscattered electron imaging; DAPI, 4',6-diamidino-2-phenylindole; EM, electron microscopy; ER, endoplasmic reticulum; F-actin, filamentous-actin; HIM, helium ion microscopy; PDI, protein disulfide isomerase; SE, secondary electron; SEM, scanning electron microscopy; SiN, silicon nitride; TEM, transmission electron microscopy

Key words: helium ion scanning microscopy, antibody, tissue, electron microscopy, ionoluminescence proteins, suggesting the formation of membrane raft structures that include membrane proteins. SE-HIM also delivered high contrast images of unstained, uncoated, thin sections of Epon-embedded mouse kidney tissues mounted on a SiN/Si bilayer, revealing the details of sub-tissues and cell organelles. A charge-coupled mechanism explaining the observed SE-HIM contrast is proposed. Ionoluminescence-HIM was also performed targeting zinc oxide particles on cells. In conclusion, the high depth-of-field, high-resolution imaging achieved using HIM may have applications in various fields, including soft materials.

\section{Introduction}

The short de Broglie wavelength of helium ions $\left(\mathrm{He}^{+}\right)$makes the resolution expected for helium ion microscopy (HIM) very high. The interaction between $\mathrm{He}^{+}$and sample atoms causes the emission of several signals that can be separately recorded (1). Several helium ion microscopes exploit secondary electron (SE) emission. Detection of the weak ionoluminescence (IL) signal was only recently reported (2) and has not yet been applied to biological samples. Based on simulations, HIM of biological samples is expected to achieve sub-nanometer resolution with efficient charge control (3). Of advantage for high resolution, the collected SEs are predicted to arise from a relatively narrow excitation volume $(4,5)$. Furthermore, the narrow convergence angle of the fine $\mathrm{He}^{+}$ beam makes the depth-of-field (DOF) great (6).

These properties indicate that HIM may be considered an important novel tool for cell biology and nanomedical research (7-9). Until recently, observation was limited to the cell surface, partly due to the metal-coating pretreatment (10) employed to reduce charging for long time-periods. However, intracellular structures have been observed by transmission HIM by using a low-brightness radio frequency ion source at a beam line faculty [accelerating voltage $1.2 \mathrm{MeV}$; (11)] rather than the standard single atom HIM source.

The present study achieved high contrast, high DOF imaging of uncoated, unstained dried cells and Epon-embedded tissue sections using the SE signal generated by a bright single atom HIM source at $30.0 \mathrm{kV}$ (SE-HIM). Labeling the cells with gold $(\mathrm{Au})$-tagged antibodies realized immuno-SE-HIM. 


\section{Materials and methods}

Cell culture and fixation. African green-monkey kidney fibroblast COS7 cells and mouse $\mathrm{C} 2 \mathrm{C} 12$ myoblast cells were either cultured directly on a silicon nitride $(\mathrm{SiN}) / \mathrm{Si}$ chip (i.e., on a $100 \mathrm{~nm} \mathrm{SiN} \mathrm{film} \mathrm{layered} \mathrm{on} \mathrm{a} 200 \mu \mathrm{m}$ Si chip) or on SiN film alone [i.e., in an atmospheric SEM (ASEM) dish, which has a SiN window supported by a Si frame in its base] (12-14). Cells were cultured in $3 \mathrm{ml}$ Dulbecco's modified Eagle's medium (Wako Pure Chemical Industries, Ltd., Osaka, Japan) supplemented with $10 \%$ fetal bovine serum (Gibco; Thermo Fisher Scientific, Inc., Waltham, MA, USA) and $100 \mu \mathrm{g} / \mathrm{ml}$ kanamycin, in an atmosphere containing $5 \% \mathrm{CO}_{2}$ at $37^{\circ} \mathrm{C}$. Following culture, cells were fixed with $1 \%$ glutaraldehyde in PBS $\left(136 \mathrm{mM} \mathrm{NaCl}, 1.4 \mathrm{mM} \mathrm{KCl}, 10 \mathrm{mM} \mathrm{Na}_{2} \mathrm{HPO}_{4}\right.$ and $1.7 \mathrm{mM} \mathrm{KH} \mathrm{PO}_{4}$; $\mathrm{pH}$ 7.4) at room temperature for $15 \mathrm{~min}$ or, if immunolabeling or fluorescence microscopy (FM) followed, with $4 \%$ paraformaldehyde in PBS ( $\mathrm{pH} 7.4$ ) at room temperature for $15 \mathrm{~min}$. Paraformaldehyde-fixed cells were immunolabeled and further fixed with glutaraldehyde. For HIM observation, fixed cells were dehydrated through an alcohol gradient series and dried.

Immunolabeling. Cells were labeled as previously described (12). Briefly, paraformaldehyde-fixed cells were perforated with 0.1 or $0.5 \%$ Triton X-100 in PBS at room temperature for $15 \mathrm{~min}$, washed with PBS and blocked with $1 \%$ skimmed milk/5\% goat serum (Gibco; Thermo Fisher Scientific, Inc.) in PBS for 20 min. For primary labeling, cells were incubated with antibodies or phalloidin in blocking solution. The antibodies used were: Mouse anti- $\alpha$-tubulin antibody (1:100 dilution in blocking solution, incubated at room temperature for $1 \mathrm{~h}$; A11126; Invitrogen; Thermo Fisher Scientific, Inc.) and rabbit anti-protein disulfide isomerase (PDI) (1:100 dilution, incubated at room temperature for $1 \mathrm{~h}$; ER Stress Antibody Sampler kit, \#9956; Cell Signaling Technology, Inc., Danvers, MA, USA). Alexa Fluor ${ }^{\circledR}$ 488-conjugated phalloidin (1:500 dilution, incubated at room temperature for $30 \mathrm{~min}$; A12379; Molecular Probes; Thermo Fisher Scientific, Inc.) or phalloidin-biotin (4 $\mu \mathrm{g} / \mathrm{ml}$; P8716; Sigma-Aldrich; Merck KGaA, Darmstadt, Germany) was used for fluorescence and fluorescence/Au-labeling of filamentous-actin (F-actin), respectively. The cells were labeled with the following secondary antibodies: Goat immunoglobulin $\mathrm{G}(\mathrm{IgG})$ against rabbit or mouse IgG conjugated with fluorescent Alexa Fluor ${ }^{\circledR} 488$ or 594 dye (1:500-1:1,000 dilution, incubated at room temperature for 30 min; A11034, A11029, A11037 and A11032; Invitrogen; Thermo Fisher Scientific, Inc.), or goat Fab' against rabbit or mouse IgG, doubly conjugated with $1.4 \mathrm{~nm}$ Nanogold $^{\circledR}$ and Alexa Fluor ${ }^{\circledR} 488$ (1:100-1:400 dilution in blocking solution, incubated at room temperature for $30 \mathrm{~min}$; NAN7204 and NAN7202; Nanoprobes, Inc., Yaphank, NY, USA). Samples labeled with phalloidin-biotin were incubated with streptavidin conjugated with Nanogold and Alexa Fluor ${ }^{\circledR} 488$ (1:50 dilution, incubated at room temperature for $30 \mathrm{~min}$; NAN7216; Nanoprobes, Inc.). To achieve counter-labeling using another type of fluorescence, samples were first incubated with rabbit anti-PDI or mouse anti- $\alpha$-tubulin antibody, then with Alexa Fluor ${ }^{\circledR}$ 594-tagged anti-rabbit or anti-mouse IgG. To achieve fluorescent labeling of nuclei, cells were stained with
$5 \mathrm{ng} / \mathrm{ml}$ 4',6-diamidino-2-phenylindole (DAPI; Sigma Aldrich; Merck KGaA) for $3 \mathrm{~min}$. For ASEM or HIM observation, the bound antibodies were fixed with $1 \%$ glutaraldehyde in PBS for $15 \mathrm{~min}$. After washing, Nanogold particles were enhanced by $\mathrm{Au}$ sedimentation using GoldEnhance EM (Nanoprobes, Inc.) at room temperature for $5 \mathrm{~min}$. For lectin labeling, paraformaldehyde-fixed COS7 cells that had been cultured on the $\mathrm{SiN} / \mathrm{Si}$ chip were blocked with $2 \%$ bovine serum albumin (Wako, Pure Chemical Industries Ltd.) in PBS for 30 min without perforation. Surface glycans on the cells were labeled with wheat germ agglutinin (WGA)-15 nm colloidal Au (EY Laboratories, Inc., San Mateo, CA, USA) at a final concentration corresponding to 0.8 at absorbance 520 for $30 \mathrm{~min}$. The labeled cells were fixed with $1 \%$ glutaraldehyde in PBS for 15 min.

Tissues. Adult mice were anesthetized with isoflurane and sacrificed to obtain kidney tissues for observation. Briefly, the kidney samples were diced, and the pieces were fixed with $2.5 \%$ glutaraldehyde in $0.2 \mathrm{M}$ phosphate buffer for $30 \mathrm{~min}$, dehydrated through a gradient series of alcohol at room temperature, embedded in Epon, and sectioned using a Leica Ultracut UCT ultramicrotome (Leica Microsystems, Inc., Buffalo Grove, IL, USA). The sections were placed on a SiN/Si chip for SE-HIM or a carbon-covered copper mesh grid for transmission electron microscopy (TEM). The animal studies were conducted in compliance with the national institutional rules of conduct of Advanced Industrial Science and Technology (AIST; Tsukuba, Japan). The present study was approved by the Animal Care and Use Committee of the National Institute of AIST.

HIM. A Carl Zeiss Orion Plus HIM (Carl Zeiss Microscopy, LLC, Peabody, MA, USA) was used in the present study. The $\mathrm{SiN} / \mathrm{Si}$ chip or ASEM dish (14) holding the cells was attached to the metal specimen holder of the HIM with conductive carbon tape (3 M; Japan Ltd., Tokyo, Japan) as illustrated in Fig. 1A left and center, respectively. Dried cells and tissues were imaged using an acceleration voltage of $30.0 \mathrm{kV}$ and a beam current of $\sim 2.0 \mathrm{pA}$. The radiation dose was $<14.4 \mathrm{He}^{+} / \AA^{2}$. The flood gun of the HIM was employed mainly in conjunction with the ASEM dish to reduce charging, and generally not with the SiN/Si chip. Use of the flood gun entailed rastering the beam of electrons over the same area as the ion beam; the electrons partially neutralize the surface charge, enabling SE to leave the surface with sufficient energy to be collected by the SE detector. Small adjustments were made to the electron beam energy, and the $\mathrm{X}$ and $\mathrm{Y}$ deflectors, during imaging to ensure that the best possible image was obtained. For correlative microscopy, labeled cells were first checked in solution using FM and/or the inverted SEM of an ASEM and, after drying, by SE-HIM.

IL-HIM. COS7 cells cultured on a SiN/Si bilayer were incubated with zinc oxide $(\mathrm{ZnO})$ nanoparticles $(\mathrm{ZnO}-\mathrm{NPs}$; Sigma-Aldrich; Merck KGaA) for $7 \mathrm{~h}$ at $37^{\circ} \mathrm{C}$. The cells were fixed with $4 \%$ paraformaldehyde as aforementioned. The cells were further fixed with glutaraldehyde, dehydrated in alcohol and air-dried, after which they were observed by IL-based HIM. Briefly, an elliptical mirror in the specimen chamber of the HIM is used to efficiently collect luminescence from the sample, and the light is transferred, separated by diffraction 


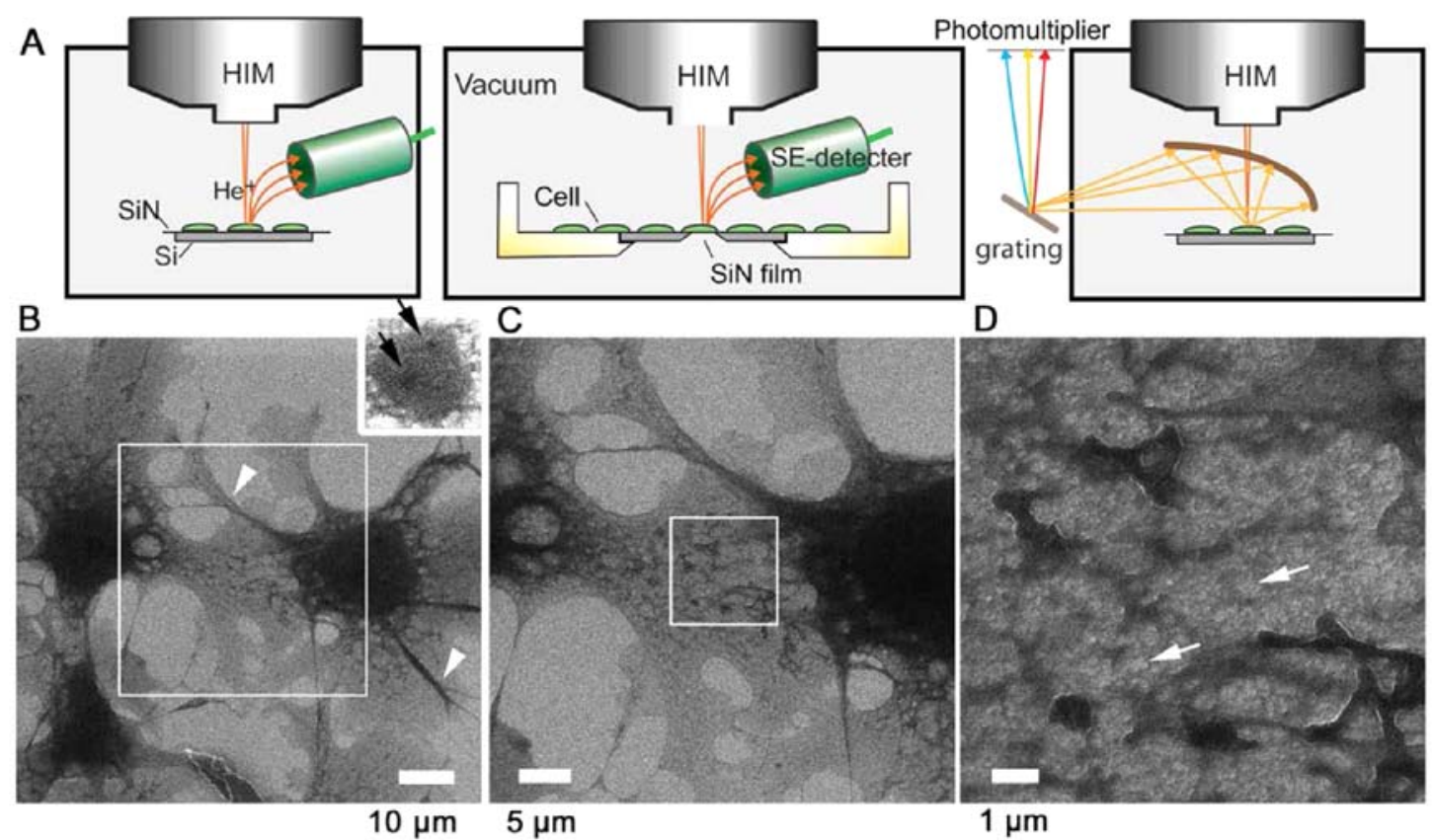

Figure 1. SE-HIM and IL-HIM of uncoated, unstained fibroblast cells. (A) Schematic diagrams. The sample is scanned by a He $\mathrm{e}^{+}$beam and the emitted SEs are detected (left and center). Alternatively, emitted IL is collected by an elliptical mirror, separated by grating and detected by a photomultiplier (right). (B) SE-HIM of uncoated, unstained COS7 cells on a SiN/Si bilayer. The background is bright; cell boundaries are clearly defined. The dark central region is the nucleus. Inset: Adjusted to visualize darker nucleoli-like structures (black arrows) within the nucleus on the right. (C) Image of the rectangle in B. Dark striated bundles radiate from the nucleus to the cell periphery. A web-like network, the ER, is prominent between them, particularly near the nucleus. (D) Image of the rectangle in C. Contrast variations indicate that the ER network is not uniformly thick. ER, endoplasmic reticulum; $\mathrm{He}^{+}$, helium ion; HIM, helium ion microscopy; IL-HIM, ionoluminescence-HIM; SE, secondary electron; $\mathrm{SiN} / \mathrm{Si}$, silicon nitride/silicon.

grating and detected by a photomultiplier (Fig. 1A, right). The light intensity is plotted for each pixel in the output image, according to the timing signal of the scan frame shift.

TEM. Unstained Epon thin-sections on carbon-covered copper EM grids were imaged using a Hitachi H7600 TEM (Hitachi, Ltd., Tokyo, Japan) at x500-2,000 magnification with an acceleration voltage of $80 \mathrm{kV}$.

ASEM and FM. The ClairScope ASEM correlative-microscope (JASM-6200; JEOL, Ltd., Tokyo, Japan) was used to record fluorescence and SEM images of the samples in solution (14) as references. The standard 8 -window $35 \mathrm{~mm}$ bio-ASEM dish with a $100 \mathrm{~nm}, 0.25 \times 0.25 \mathrm{~mm}$ SiN film window built into its base (JEOL, Ltd.) was employed for cell culture (13). After fixation and labeling, cells in PBS were observed with the fluorescence microscope of the ASEM equipped with a 40x objective lens (NA: 0.8), or with an Olympus-AX fluorescence microscope equipped with a $60 x$ objective lens (NA: 1.1). After Au enhancement, the medium was supplemented with $10 \mathrm{mg} / \mathrm{ml}(\mathrm{w} / \mathrm{v})$ ascorbic acid as a radical scavenger, and the cells were directly observed in buffer using the inverted SEM of the ASEM at an acceleration voltage of $30 \mathrm{kV}$; the backscattered electrons were recorded.

\section{Results}

SE-HIM of fibroblast cells on a SiN/Si bilayer. Unstained and uncoated African green monkey COS7 kidney fibroblast cells cultured on a $\mathrm{SiN} / \mathrm{Si}$ substrate were fixed, dried and observed in vacuum by SE-HIM without counter electron-radiation (Fig. 1A, left). Cells appeared dark on a bright background from the substrate, and the nucleus and delicate intracellular structures were easily distinguishable (Fig. 1B). The nucleus displayed even darker nucleoli-like structures (Fig. 1B inset, arrows). While the lamellipodia were relatively homogeneous (Fig. 1B and C), medium-dark striated bundles including actin stress fibers or microtubules radiated from the region around the nucleus to the cell periphery (Fig. 1B, arrowheads) and were present in most filopodia. The dark web-like network prominent between these bundles, particularly near the nucleus (Fig. 1C), corresponds to the ER. The ER extended throughout the cytoplasm (Fig. 1D) and surrounded gray cytoplasmic structures and various brighter features (Fig. 1D, arrows) that might be vacuoles. The white line at the edge of the ER may arise from enhanced SE emission (Fig. 1D).

These observations were confirmed by correlative FM (Fig. 2). Cells were labeled with fluorescent cell organelle markers for DNA (Fig. 2A), PDI (Fig. 2B), which is present in the ER lumen, and F-actin (Fig. 2C). The fluorescence signals from the ER (Fig. 2B, arrows) correspond to thick web-like networks in the SE-HIM images (Fig. 2E, arrows), whereas the fluorescence signals from F-actin (Fig. 2C, arrowheads) correspond to the faint striated bundles in the SE-HIM images (Fig. 2E, arrowheads).

Stress fibers in C2C12 myoblast cells. F-actin and PDI in C2C12 myoblast cells were similarly labeled and observed by FM (Fig. 3A-D) and SE-HIM (Fig. 3E-H). Myoblasts possess abundant stress fibers. The nucleus and sarcoplasmic reticulum (SR) were the darkest regions observed on the HIM 


\section{FM}
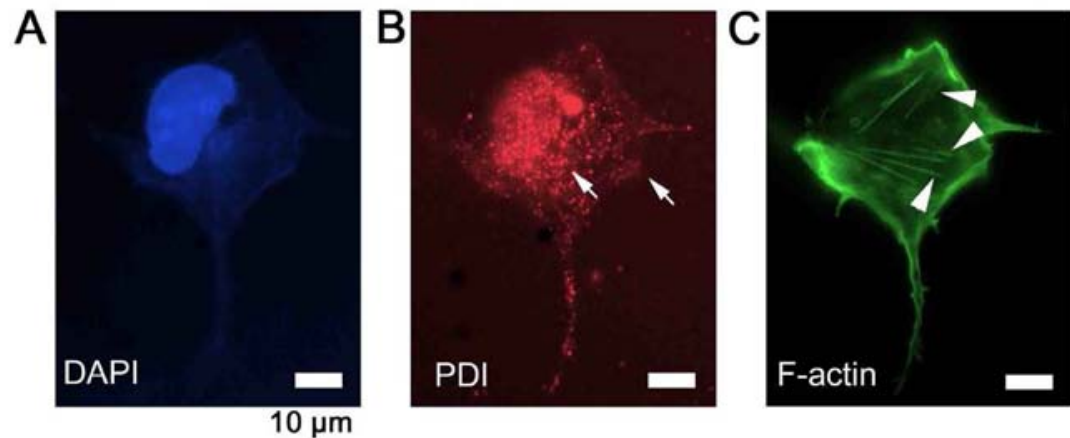

HIM
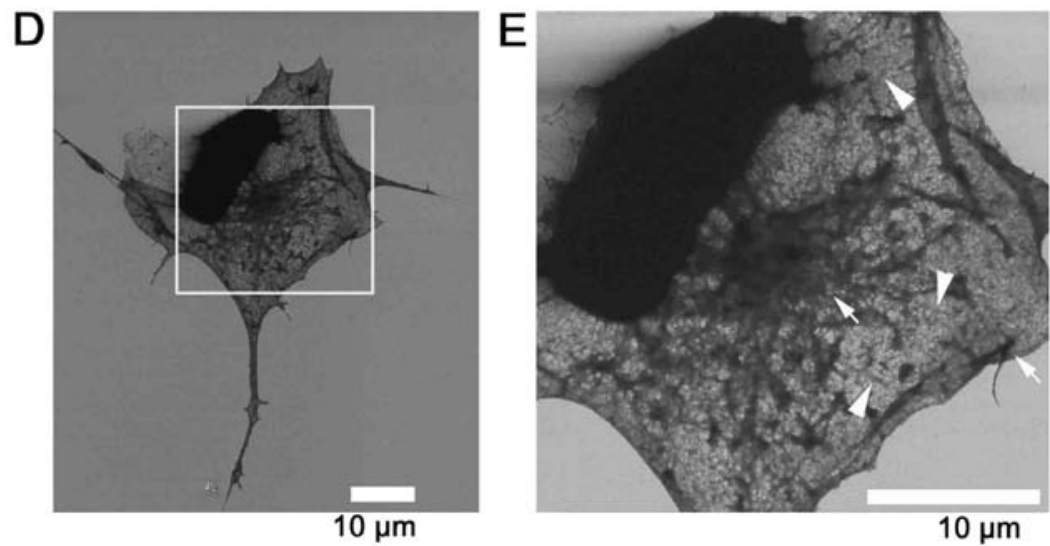

Figure 2. Fluorescence identification of organelles imaged by SE-based HIM. To identify the organelles of COS7 cells, F-actin was labeled with Alexa Fluor ${ }^{\circledR} 488$-conjugated phalloidin (green), ER with anti-PDI antibody and further with Alexa Fluor ${ }^{\circledR}$ 594-conjugated secondary antibody (red), and DNA with DAPI (blue). Labeling was checked in solution by fluorescence microscopy: (A) DAPI was used to identify the nucleus. (B) PDI was used to identify the ER. (C) F-actin was used to identify stress fibers. Cells were then dehydrated/dried and observed by SE-HIM. (D) SE-HIM, corresponding to C. (E) Magnification of the rectangle in D. The web-like network in the SE-HIM images corresponds to florescence signals from the ER (arrows), and the faint striated bundles to fluorescence signals from F-actin (arrowheads). DAPI, 4',6-diamidino-2-phenylindole; ER, endoplasmic reticulum; F-actin, filamentousactin; HIM, helium ion microscopy; PDI, protein disulfide isomerase; SE, secondary electron.
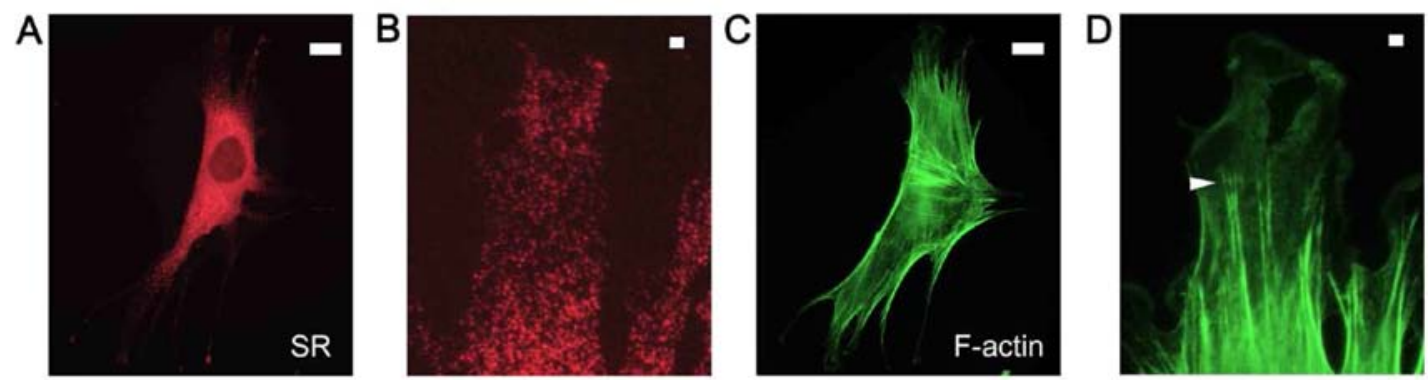

$\mathrm{E}$

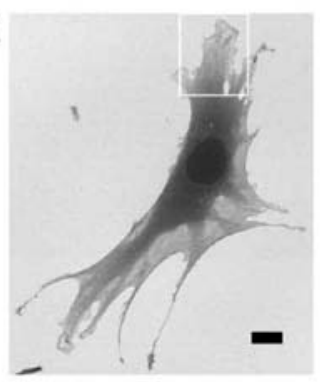

$\mathrm{F}$

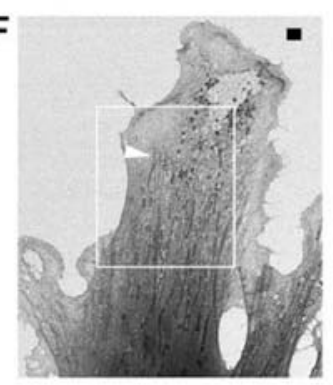

- G

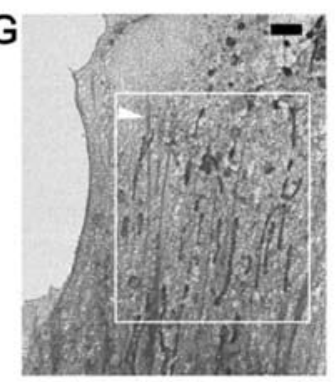

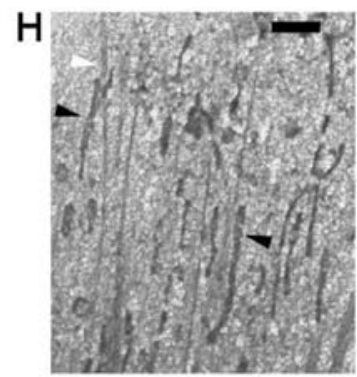

Figure 3. SR and actin stress fibers of $\mathrm{C} 2 \mathrm{C} 12$ myoblast cells visualized by correlative SE-HIM. (A and B) FM to visualize PDI (red) and localize the SR (C and D) FM to visualize F-actin (green) and reveal stress fibers; (B and D) are corresponding higher magnification images of A and C, respectively. (E-H) SE-HIM images of C. The darker region surrounding the cell nucleus is the SR. (F-H) Higher magnification images of the areas marked in the preceding figure. The darkly contrasted bundles (white arrowheads) striating the cytoplasm are F-actin. The elongated darker vesicles beside them (black arrowheads) might be mitochondria. The fainter web-like density compares well to peripheral fluorescent SR regions in B. Scale bars: $20 \mu \mathrm{m}$ in A, C and E and $2 \mu \mathrm{m}$ in B, D and F-H. F-actin, filamentous-actin; FM, fluorescence microscopy; HIM, helium ion microscopy; PDI, protein disulfide isomerase; SE, secondary electron; SR, sarcoplasmic reticulum. 


\section{Culture substrate}

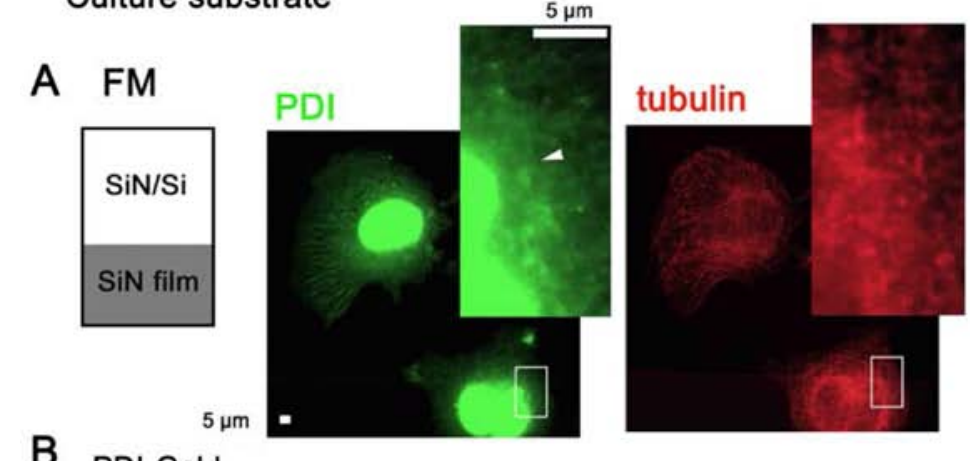

\section{B PDI-Gold}

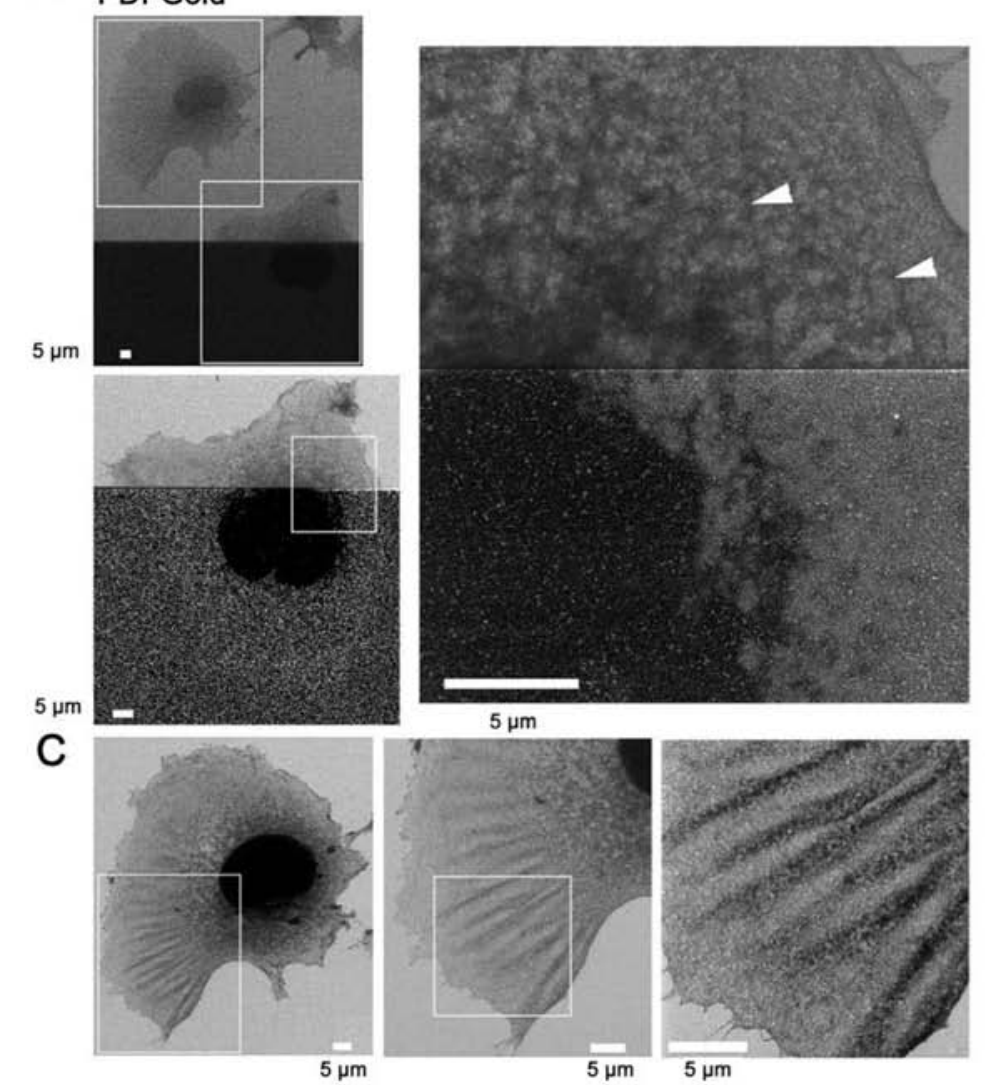

Figure 4. SE-HIM of immunogold-labeled PDI in COS7 cells on SiN film and SiN/Si. PDI was tagged with fluorescence/Au to directly identify the ER by SE-HIM, whereas $\alpha$-tubulin was labeled only with fluorescence. Cells were cultured on the electron-transparent SiN film window of an atmospheric scanning electron microscopy dish, fixed and perforated. PDI and $\alpha$-tubulin were then immunolabeled with Alexa Fluor ${ }^{\circledR} 488$-Nanogold and Alexa Fluor ${ }^{\circledR} 594$, respectively. Labeling was confirmed in buffer by correlative FM. (A) FM of PDI and $\alpha$-tubulin. Insets: Enlarged views of the white rectangles. (B) SE-HIM of PDI in cells at the border between SiN film and SiN/Si-bilayer. Bottom panel: Higher magnification image of the lower rectangle in the upper panel. Right, further magnification. In both cases, the brightness and contrast of the lower SiN film region was adjusted to make the signals visible. On SiN/Si, Au signals were present but indistinct. On SiN film, the nuclei and thick ER were speckled with bright Au signals. Filamentous density (arrowheads) visualized on the SiN/Si bilayer, was barely visible on SiN film. (C) Cell on the SiN/Si region. Left, higher magnification image of the top rectangle in the upper left panel of B. The cell has a dense web-like structure around the nucleus that is connected to a fin-like structure in lamellipodia; both structures correspond to ER fluorescence in A. Center and right, higher magnification image of the rectangle in the preceding panel. Au signals are present but indistinct. Au, gold; ER, endoplasmic reticulum; FM, fluorescence microscopy; PDI, protein disulfide isomerase; SE-HIM, secondary electron-helium ion microscopy; SiN/Si, silicon nitride/silicon.

images (Fig. 3A and 3E). The thick spindle-shaped cell body and well-developed SR exhibited darker SE signals than the corresponding regions in COS7 cells (Figs. 1 and 2). Moderately dense, 50-200 $\mathrm{nm}$ wide F-actin fibers that aligned with the cell boundary were prevalent in the cytoplasm at the cell periphery (Figs. 3C, D, F and G) and fork-shaped stress fiber bundles can be distinguished (Fig. 3D and F-H, white arrowheads). The darker, $200 \mathrm{~nm}$ wide and 1-10 $\mu \mathrm{m}$ long, vesicle-like structures present along the bundles (Fig. $3 \mathrm{H}$, black arrowheads) were considered to be mitochondria, and the fine web of material surrounding them may be a fine network of SR (Fig. 3B) or protein complexes.

HIM of immuno-Au-labeled PDI on SiN film. To directly identify the ER of COS7 cells by SE-HIM, PDI was labeled with antibodies tagged with both fluorescence and Au. The cultured cells extended over the thin SiN film window and SiN/Si chip in the base of an ASEM dish (Fig. 1A, center). Labeling was detected by FM (Fig. 4A, center and right). Cells on $\mathrm{SiN} / \mathrm{Si}$ regions (Fig. 4A, left) were clearly visible by SE-HIM, but 
FM

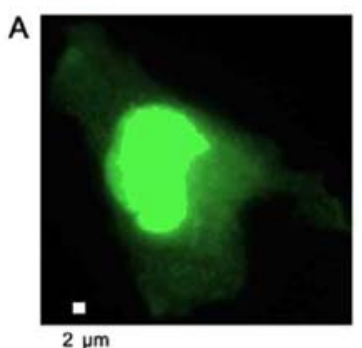

HIM

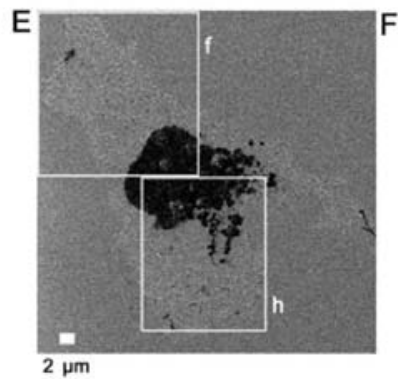

ASEM

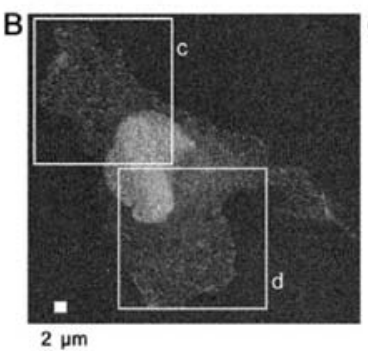

$2 \mu \mathrm{m}$

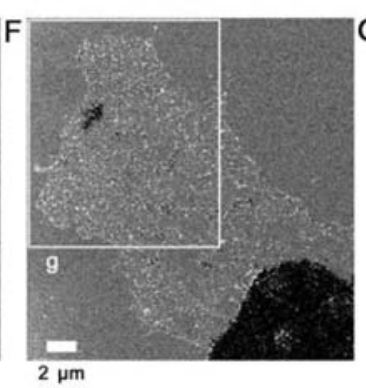

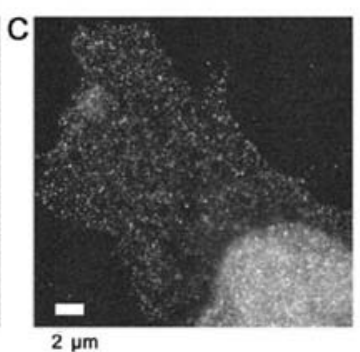

$2 \mu \mathrm{m}$

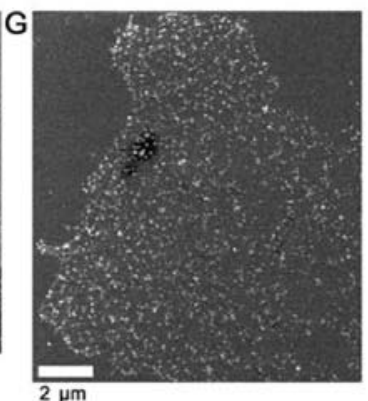

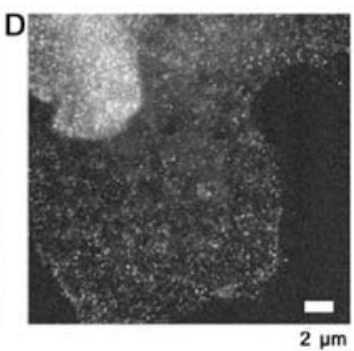

$2 \mu \mathrm{m}$

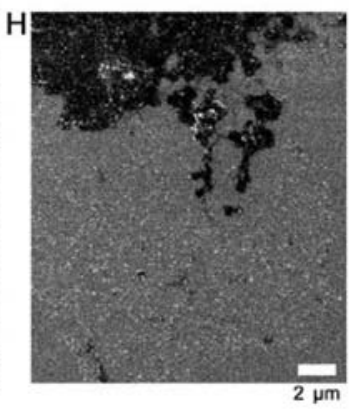

Figure 5. Au-labeled ER in cells on SiN film. COS7 cells on an ASEM dish were labeled as in Fig. 4 to directly identify the ER by SE-HIM and ASEM, and access SE-HIM imaging of Au sediments. Labeling was confirmed in buffer by correlative FM, and further by ASEM. (A) FM of PDI. (B) ASEM of the same region. Cells in buffer were scanned from underneath, the electron beam passing through the SiN film, and backscattered electrons were detected. The high scattering centers (white) are Au sediments. (C) Image of rectangle c in B. (D) Image of rectangle d in B. Afterwards, cells were dehydrated/dried and observed by SE-HIM. (E) SE-HIM of the cell imaged in A and B. Both the nucleus and the thick ER are very dark. (F) Image of rectangle $f$ in E. (G) Image of rectangle $g$ in F. Au signals evident as white dots, form a web-like pattern over the cytoplasm and also cover parts of the nucleus, but to a lesser extent than indicated by ASEM. (H) Image of rectangle $\mathrm{h}$ in E. The contrast of the ER seems to be related to its diameter. In the SE-HIM image, the concentration of Au signals in thin ER regions is higher than in thick ER regions. The sum of the bright (Au) and the dark signals in the SE-HIM image matches the ASEM. ASEM, atmospheric scanning electron microscopy; Au, gold; ER, endoplasmic reticulum; FM, fluorescence microscopy; PDI, protein disulfide isomerase; SE-HIM, secondary electron-helium ion microscopy; SiN, silicon nitride.

scattering from the Au sediments (bright spots) was difficult to distinguish (Fig. 4C). On SiN film regions (Fig. 4A, left), $\mathrm{Au}$ signals could be clearly visualized (compare Fig. 4B, bottom left and right, with gain adjustment, and Fig. 4B, upper left, without adjustment). Moderately dark filamentous features on the SiN/Si bilayer (Fig. 4B, arrowheads) were not visible on the SiN-film. Similarly, although the nucleus and thick ER were very dark on SiN film only faint traces of the thinner ER present at the cell periphery could be distinguished (Fig. 4B). Notably, both ER regions were similarly speckled by Au signals (Fig. 4B); only the contrast and degree of PDI fluorescence reflected their thickness (Fig. 4A). Other COS7 cells cultured on an ASEM dish were fixed with paraformaldehyde and similarly labeled. Afterwards, cells on the SiN film were observed in radical scavenger glucose solution by ASEM (Fig. 5A-D). The cells were then dehydrated and observed in a vacuum by SE-HIM (Fig. 5E-H). The SE-HIM Au signal corresponded to the Au signal recorded by ASEM (Fig. 5B-D), which is comparable to the signal obtained by typical immuno-EM (15).

Au-labeled F-actin and HIM image resolution. The F-actin of $\mathrm{C} 2 \mathrm{C} 12$ cells was tagged with fluorescence and $\mathrm{Au}$, inspected for labeling by FM (Fig. 6A) and ASEM (Fig. 6B), and imaged by SE-HIM (Fig. 6C). Au signals from labeled F-actin were visible in the HIM images, regardless of whether the substrate was SiN-film or SiN/Si (Fig. 6C). They were highly concentrated towards the edge of lamellipodia, moderately present on the internal mesh-like F-actin network (Fig. 6C, right) and also visible on filopodia (Fig. 6C, arrowhead). This distribution agrees with FM (Fig. 6A) and ASEM (Fig. 6B), particularly at thin cell margins, thus indicating that SE-HIM captures images of regions close to the cell surface. $\mathrm{C} 2 \mathrm{C} 12$ cells cultured on the SiN/Si chip (Fig. 1A, left) were similarly labeled to measure the resolution. From the minimum recognizable gap between two Au particles, the resolution was $2 \mathrm{~nm}$ (Fig. 6D).

All three types of microscopy clearly revealed actin stress fibers in the cell bodies (Fig. 7B, H and M, arrows and arrowheads) and filopodia (Fig. 7B, C, I, J and L, O-R) of correspondingly labeled COS7 cells. Actin fibers that passed below nuclei, which were imaged from below in solution by ASEM (Fig. 7H, arrowheads), were not visible from above by SE-HIM (Fig. 7M); the observable specimen thickness of ASEM is $2-3 \mu \mathrm{m}$ from the $\mathrm{SiN}$ film surface, whereas the height of COS7 cells usually exceeds $7 \mu \mathrm{m}$ in solution.

Au-labeled tubulin. Tagging the $\alpha$-tubulin of COS7 cells with fluorescence alone (Fig. 8), and fluorescence and Au (Fig. 9) allowed microtubules to be identified and distinguished from the ER visualized by SE-HIM. In the fluorescence-labeled cells on the SiN/Si substrate, FM and SE-HIM indicated that the distribution of microtubules largely overlapped with the ER (Fig. 8). Differences were mainly evident towards the cell periphery, allowing some moderately-dark fibers on the SE-HIM images (Fig. 8F, arrowheads) to be assigned as microtubules (Fig. 8C, arrowheads). In the dually labeled cells on $\mathrm{SiN}$ film, microtubules appeared as lines of fine 

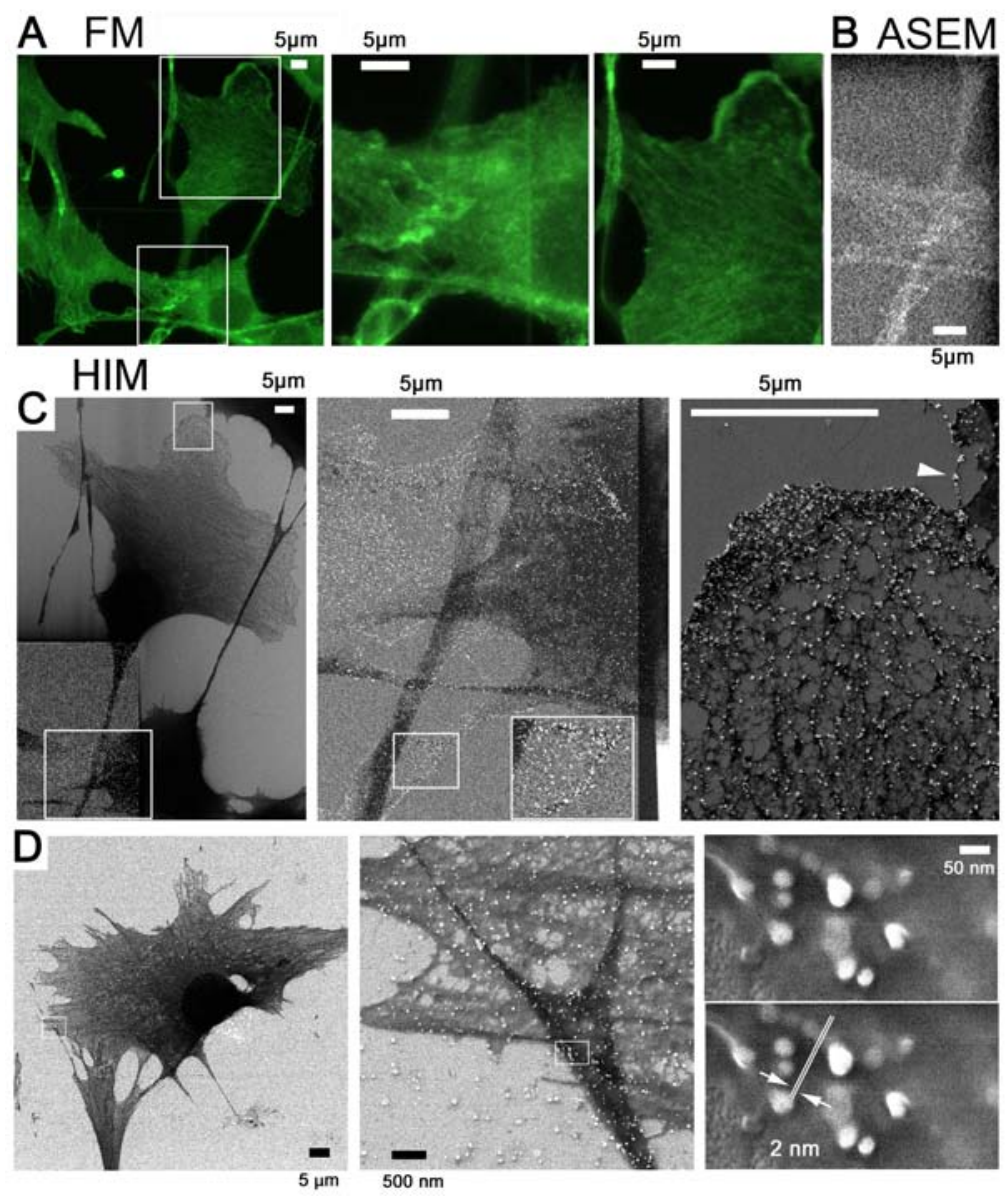

Figure 6. Au-labeled F-actin in C2C12 cells on SiN film and SiN/Si bilayer substrates, and the resolution of SE-HIM. (A-D) F-actin in C2C12 cells was tagged with fluorescence/Au. (A-C) Cells were cultured in an ASEM dish and imaged on the SiN film and SiN/Si-bilayer. (A) Fluorescence microscopy; center and right, corresponding enlarged views. (B) Au visualized by ASEM; compare A center panel. (C) SE-HIM; compare A and B. Cells and their thin lamellipodia were dark, particularly on the $\mathrm{SiN}$ film at the bottom left (brightness and contrast adjusted to make the signal visible). Center and right, higher magnification images of the marked areas. Au signals can be discerned along filamentous structures in the cytoplasm and in the lamellipodia, as well as in the philopodia (white arrowhead) of cells on the SiN/Si bilayer, on the F-actin network and at the edge of the cell. (D) Resolution was measured using similarly labeled cells cultured on a SiN/Si chip. Au was imaged at various magnifications by SE-HIM. Left, 1,143x; center, 22,860x; right, 228,600x; the measurement indicated in the lower of the two otherwise identical panels in the right frame showed the resolution to be $2 \mathrm{~nm}$. ASEM, atmospheric scanning electron microscopy; $\mathrm{Au}$, gold; F-actin, filamentous-actin; SE-HIM, secondary electron-helium ion microscopy; SiN/Si, silicon nitride/silicon.

white dots (Fig. 9G-J); the signals were less clear on $\mathrm{SiN} / \mathrm{Si}$ substrate (Fig. 9G, left). Some features observed by ASEM were not evident on the HIM images, whereas others were clearly revealed (Fig. 9C-J).

WGA-15 nm colloidal Au-labeled COS7 cells. Non-permeabilized COS7 cells were labeled with WGA-Au conjugate and imaged by SE-HIM (Fig. 10). Some of the Au particles on the surface of the cells were clustered, forming patches $\sim 50-200 \mathrm{~nm}$ in diameter. This might represent the gathering of glycosylated proteins, suggesting the presence of membrane raft structures that include membrane proteins. Again, $2 \mathrm{~nm}$ resolution was achieved.

IL-HIM. IL-HIM was developed to realize multicolored labeling/imaging that is detectable regardless of the cellular structures above epitopes. Fixed COS7 cells with ZnO-NPs were examined on a SiN/Si support. Signals corresponding to the ZnO-NPs were clearly detected above very little cell background (Fig. 11). Until recently (2) the SE emission caused by a $\mathrm{He}^{+}$beam was considered too little to yield detectable electron excitation-triggered fluorescence.

Epon-embedded tissue sections. High contrast images were recorded from unstained, uncoated thin sections of mouse kidney tissue embedded in Epon by SE-HIM, revealing detailed features of sub-tissues and cell organelles (Fig. 12A and B). The contrast was sometimes the inverse of that delivered by TEM (Fig. 12C and D) and also of SE-HIM images of dehydrated/dried cells (Figs. 1-10). In the latter case this was due to the presence of Epon; Epon-filled spaces are dark in SE-HIM images (Fig. 12A and B).

\section{Discussion}

The present study was designed to observe uncoated and unstained cells by HIM (16) and gain information about the biological systems. The results indicated that the lack of 


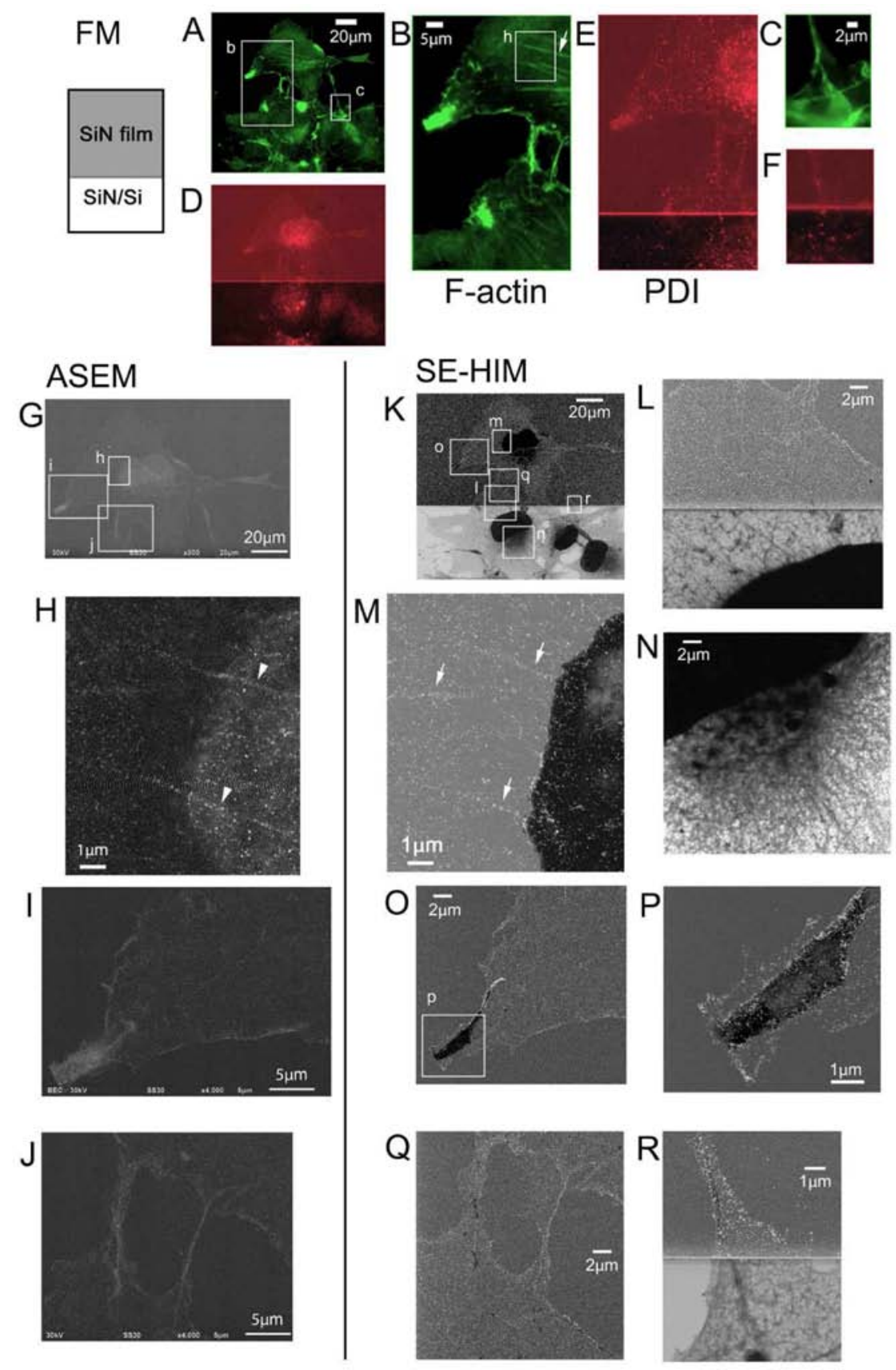

Figure 7. Au-labeled F-actin in COS7 cells. F-actin and PDI were tagged with Alexa Fluor ${ }^{\circledR} 488$ fluorescence/Au and Alexa Fluor ${ }^{\circledR} 594$, respectively. The cells were close to or across the boundary between the SiN/Si-bilayer and SiN film of the ASEM dish. The influence of the substrate can be discerned. (A-C) FM of F-actin. (A) Larger field. (B) Enlarged view of b in A. (C) Enlarged view of c in A. (D-F) FM of PDI; same fields as A, B and C, respectively. (G-J) ASEM. (G) The cell imaged in the upper region of A. (H) Enlarged image of h in G. (I) Enlarged image of $i$ in G. (J) Enlarged image of j in G. (K-R) SE-HIM. (K) Image corresponding to A. (L-R) Enlarged images of rectangles l-r in K and of the rectangle p in O, respectively. Au signals can be clearly distinguished, particularly on the SiN film. In the ASEM and SE-HIM images, signals from Au-labeled F-actin formed rows indicating the presence of various filaments in the cytoplasm. (H and M) Regions of actin fibers passing below the nuclei and imaged from below by ASEM (arrowheads), were not visible from above by SE-HIM. ASEM, atmospheric scanning electron microscopy; Au, gold; F-actin, filamentous-actin; PDI, protein disulfide isomerase; SE-HIM, secondary electron-helium ion microscopy; $\mathrm{SiN} / \mathrm{Si}$, silicon nitride/silicon.

intracellular details in SE-HIM images of uncoated cells in a previous study (10) is associated with the electrical conductivity of the sample, substrate and surroundings.

The SiN/Si substrate provided a backlight effect on SE-HIM images, thus facilitating the visualization of cytoskeletal protein complexes and intracellular membrane systems in dried cells. Variations in ER thickness were detectable, and the clarity with which mitochondria were imaged may allow the study of mitochondria-associated diseases and apoptosis.
Notably, the cytoskeleton that was quite prominent on $\mathrm{SiN} / \mathrm{Si}$ supports readily became invisible on $\mathrm{SiN}$ film, which provided less backlight.

The visibility of Au labels employed in immuno-SE-HIM was associated with both the substrate and the structure covering the $\mathrm{Au}$. The reduced background signal from SiN film allowed the Au sediments labeling F-actin, tubulin and the ER to be clearly detected. Detection was more difficult on $\mathrm{SiN} / \mathrm{Si}$ due to the bright background, and depended on where 


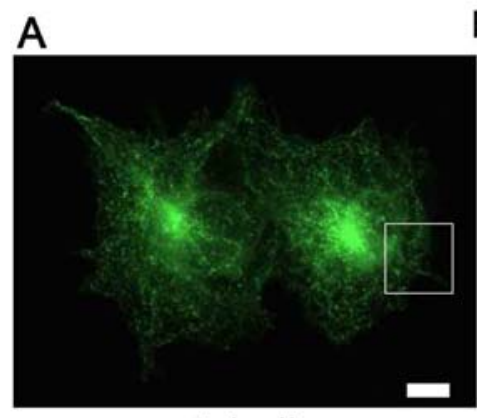

$\alpha$-tubulin
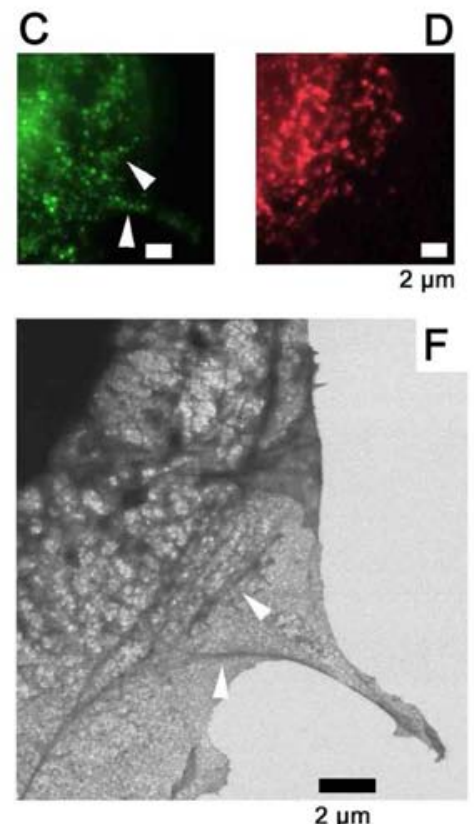

FM
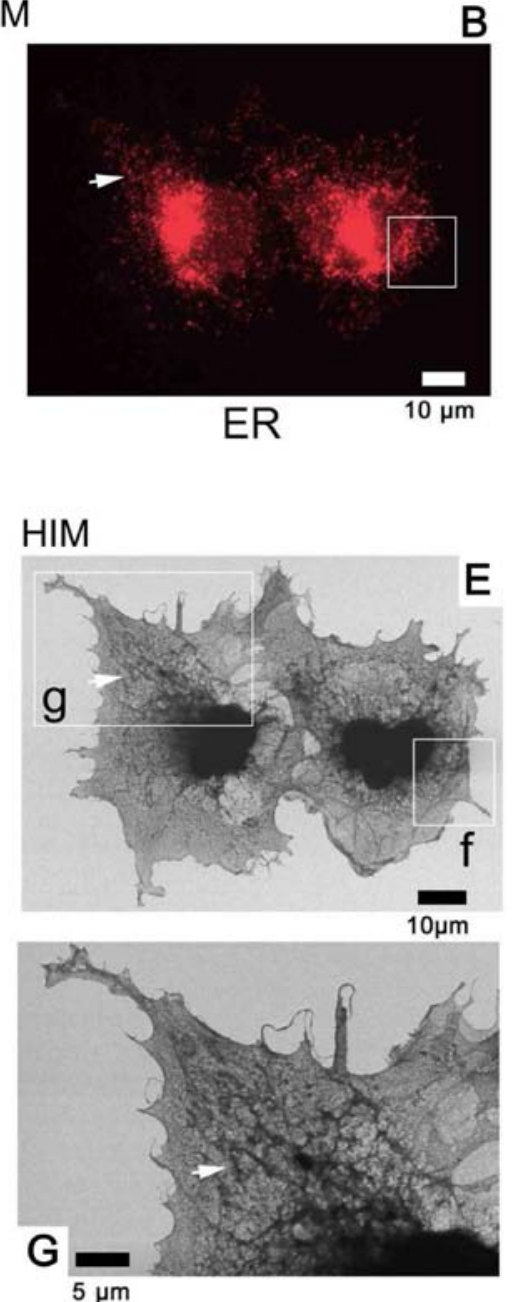

Figure 8. Use of fluorescence to identify microtubules. To identify other bundle-like densities in COS7 cells cultured on SiN/Si substrate, $\alpha$-tubulin and PDI were tagged with Alexa Fluor ${ }^{\circledR} 488$ and Alexa Fluor ${ }^{\circledR}$ 594, respectively, and observed by FM and further by SE-HIM. (A) FM of $\alpha$-tubulin. (B) FM of PDI in the same region. (C) Enlarged view of the rectangle in A. (D) Enlarged view of the corresponding rectangle in B. (E) SE-HIM, corresponding to A. (F) Image of rectangle $\mathrm{f}$ in E. (G) Image of rectangle $\mathrm{g}$ in E. Both FM and SE-HIM indicated that the distribution of microtubules largely overlapped the endoplasmic reticulum. Differences are mainly evident towards the cell periphery, allowing some moderately-dark fibers on the SE-HIM images (F, arrowheads) to be assigned as microtubules: Compare $\mathrm{E}$ and $\mathrm{G}$ with $\mathrm{A}$ and $\mathrm{B}$, arrows indicate features that are also recognizable in PDI fluorescence images; compare $\mathrm{F}$ with $\mathrm{C}$ and $\mathrm{D}$, arrowheads indicate features that are also recognizable in the $\alpha$-tubulin fluorescence images. FM, fluorescence microscopy; PDI, protein disulfide isomerase; SE-HIM, secondary electron-helium ion microscopy

the labeled structure was localized in the cell. As it is ubiquitously distributed, clear signals from F-actin were always observed, but not from the relatively internal microtubules and ER. Notably, signals were registered from an F-actin filament situated close to the cell bottom, i.e., the DOF of SE-HIM and the thickness of the dried cell were comparable.

Uncoated, unstained thin kidney tissue sections were imaged with unprecedented clarity by SE-HIM. The contrast was often the inverse of the contrast delivered by TEM. TEM images basically reflect the electron density and mass thickness of the sample.

According to Monte-Carlo simulations, the $\mathrm{He}^{+}$beam penetrates a $\mathrm{Si}$ substrate to a depth of a few hundred nanometers $(4,6)$ (Fig. 13) and the escape depth of the generated SEs is 5-10 nm. However, SE-HIM of uncoated, unstained cells on $\mathrm{SiN} / \mathrm{Si}$ or $\mathrm{SiN}$ film revealed structures at greater depth, thus suggesting that the $\mathrm{He}^{+}$beam penetrates at least the cell and a $100 \mathrm{~nm} \mathrm{SiN} \mathrm{film.} \mathrm{Two} \mathrm{effects} \mathrm{contribute} \mathrm{to} \mathrm{the} \mathrm{observed}$ contrast: (i) SE emission related to atomic number and density of the sample surface (5-10 nm depth) and (ii) emission blockage by the positive charges accumulating due to the $\mathrm{He}^{+}$beam; the degree will depend on the electrical conductivity of the material. Like SiN, Epon resin is an insulator; when uniformly thin sections mounted on $\mathrm{SiN} / \mathrm{Si}$ are irradiated by $\mathrm{He}^{+}$, positive charges will accumulate, and preclude SE emission from the surface. Although the possibility of SE emission directly from within the DOF still cannot be entirely excluded, the pixel darkness in the images may primarily reflect the degree of blockage, which will be decreased by electron-conducting components (Fig. 13B, green). Contrast variations arising from surface density variations are considered comparatively small, as the density of Epon resin is similar to that of lipids and proteins. Accordingly, the bright intensity of red blood cells is probably explained by the conductive nature of iron-associated proteins.

The inverse contrast of dried cells on $\mathrm{SiN} / \mathrm{Si}$ is attributable to differences in the substance surrounding the cell components (vacuum vs. Epon) and to the thickness of the cells. Most charges generated in the thin insulating SiN layer 


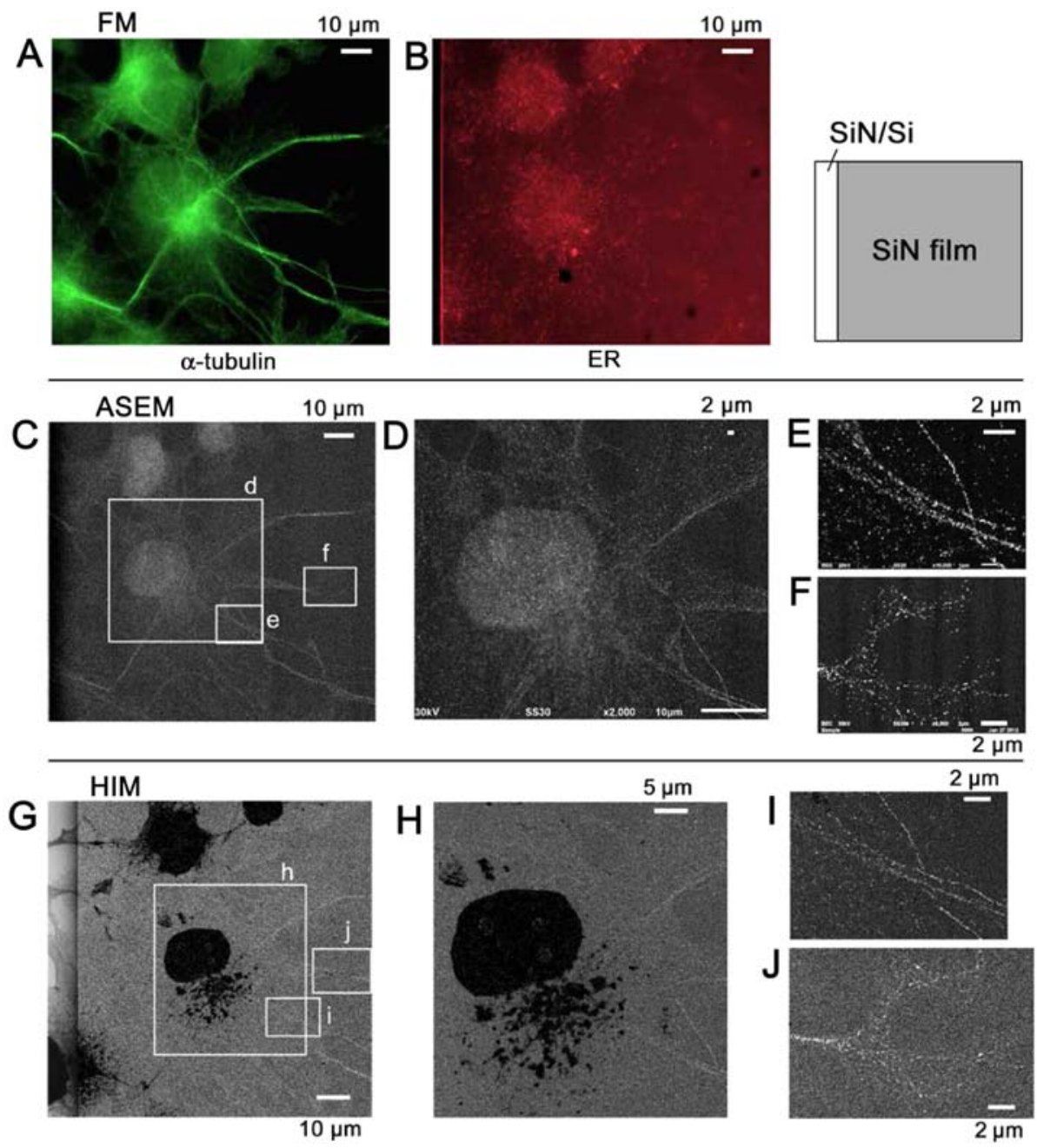

Figure 9. Immuno-SE-HIM of $\alpha$-tubulin in COS7 cells. The use of Au labels and SE-HIM is essential to distinguish the microtubules of COS7 cells. $\alpha$-tubulin and PDI in COS7 cells cultured on an ASEM dish, were tagged with Alexa Fluor ${ }^{\circledR}$ 488-Nanogold and Alexa Fluor ${ }^{\circledR}$ 594, respectively. After FM and confirmation of Au labeling by ASEM, dehydrated/dried cells were observed by SE-HIM. (A) FM of $\alpha$-tubulin. (B) FM of PDI in the same region. (C) ASEM, almost corresponding to A. (D) Image of rectangle $d$ in C. (E and F) Image of rectangles e and $f$ in C, respectively. (G-J) SE-HIM. (G) SE-HIM corresponding to A; the boundary between the $\mathrm{SiN} / \mathrm{Si}$ bilayer and the $\mathrm{SiN}$ film can be seen. The nucleus and endoplasmic reticulum are very dark, but there are still bright filaments in the cytoplasm; compare C. (H) Image of rectangle h in G. (I) Image of the rectangle i in G. The filamentous lines are comprised of white dots, i.e., signals from Au sedimentation. (J) Image of rectangle $\mathrm{j}$ in G. Microtubules in filopodia were visualized by this method. The SE-HIM and ASEM images appear similar, with the exception of the inversely contrasted organelle. ASEM, atmospheric scanning electron microscopy; Au, gold; FM, fluorescence microscopy; PDI, protein disulfide isomerase; SE-HIM, secondary electron-helium ion microscopy; SiN/Si, silicon nitride/silicon.

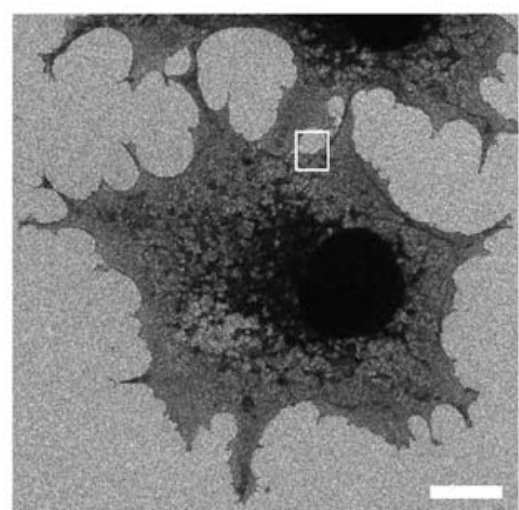

$10 \mu \mathrm{m}$

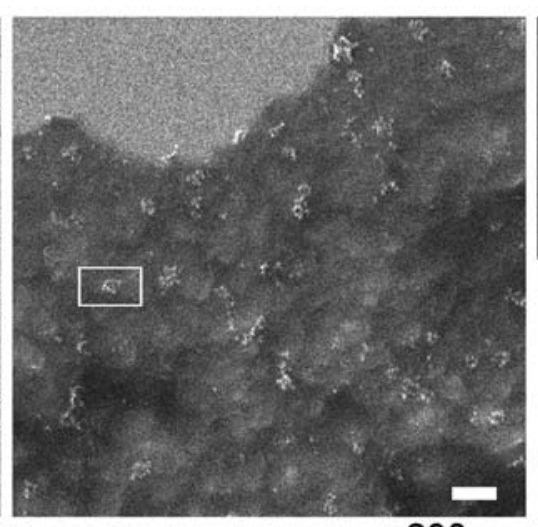

$200 \mathrm{~nm}$
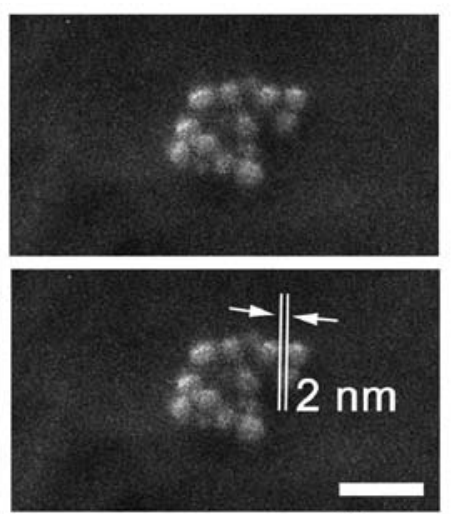

$50 \mathrm{~nm}$

Figure 10. WGA-colloidal gold-labeled surface glycan of COS7 cells. Surface glycans were labeled with WGA-colloidal Au conjugates (15 nm in diameter) on COS7 cells cultured on a SiN/Si chip. Au was imaged at various magnifications by SE-HIM. Clusters of WGA-Au particles $\sim 50-200 \mathrm{~nm}$ in diameter were observed on COS7 cells. These might represent the gathering of glycosylated proteins, suggesting the presence of membrane raft structures that include membrane proteins. Left, 1,524x; center, 45,720x; right, 228,600x; the measurement indicated in the lower of the two otherwise identical frames of the right panel showed the resolution to be $2 \mathrm{~nm}$. Au, gold; SE-HIM, secondary electron-helium ion microscopy; SiN/Si, silicon nitride/silicon; WGA, wheat germ agglutinin. 


\section{SE-HIM}

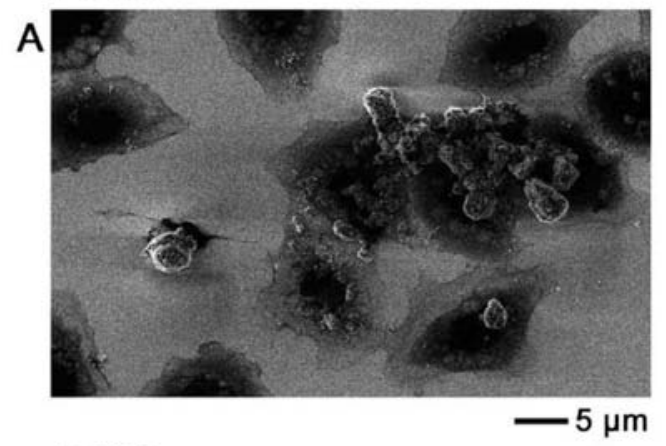

\section{IL-HIM}
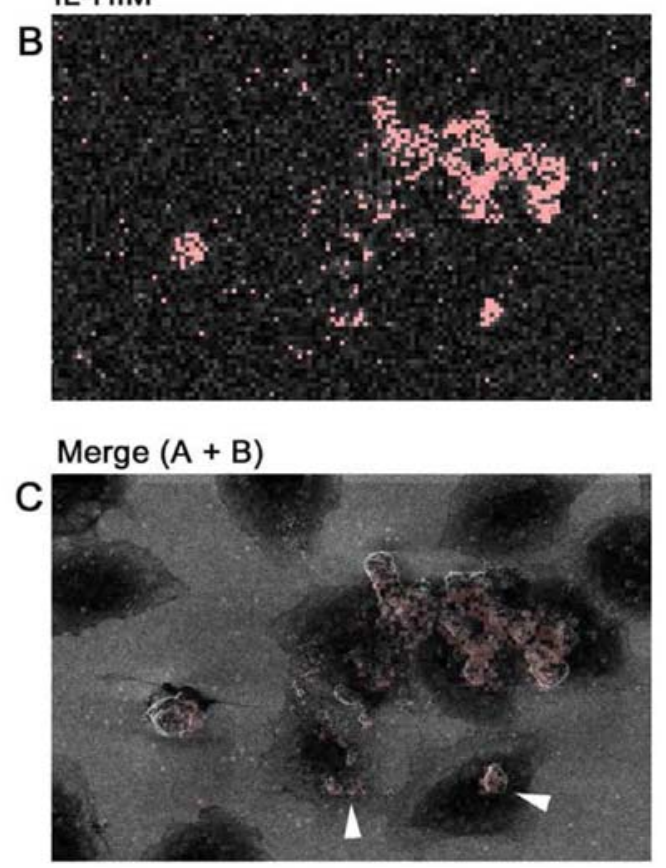

Figure 11. IL-HIM. Fixed COS7 cells that had been cultured with ZnO-NPs were imaged on silicon nitride/silicon substrate by (A) SE-HIM and (B) IL-HIM. (C) Overlay of the IL-HIM image (red) on the SE-HIM image. ZnO-NPs were visualized (arrowheads). HIM, helium ion microscopy; IL, ionoluminescence; $\mathrm{SE}$, secondary electron; ZnO-NPs, zinc oxide-nanoparticles.

will be directly dissipated by the underlying $\mathrm{Si}$; SE emission will not be inhibited, and the corresponding image regions will be bright. Being insulators like Epon (17), phospholipid membranes will partially block SE emission, i.e., cells will be comparatively dark. The contrast of very thin filopodia or lamellipodia will be less as the $\mathrm{He}^{+}$beam is primarily scattered in the substrate (Fig. 13C). In the middle of the cell, the $\mathrm{He}^{+}$beam is mainly scattered in the thick cell body (Fig. 13D). Structures with higher density and large atomic number scatter more, creating more positive charges, which further accumulate as the underlying intracellular lipid membrane inhibits their dissipation. These regions are therefore darker on the images.

SE-HIM images of SiN film alone and of the cells on it, are comparatively dark. This is because the insulator film is not lined by a conductor; positive charges formed by $\mathrm{He}^{+}$irradiation easily accumulate precluding SE emission (Fig. 13E) and the effect is more pronounced when a cell is present (Fig. 13F). However, unless they were covered by large membranous cell organelles, Au sediments were clearly visible. Since Au is a conductor, it can efficiently dissipate accumulated charge to the surroundings. This will alleviate charging near the surface and facilitate SE emission.

The contrast of SE-HIM might be related to the "voltage contrast' observed when insulator or insulator semiconductor composites are imaged by SE-SEM (18). Voltage contrast is affected by the dielectric constant of the irradiated material and by the beam-induced current (conductivity) (18). These factors might also influence the contrast of SE-HIM in the present study, although the electron beam used for SE-SEM is substituted by a $\mathrm{He}^{+}$beam in SE-HIM.

The $2 \mathrm{~nm}$ resolution obtained for labeled-Au particles is one of the most attractive features of HIM. However, in immunolabeling experiments, the localization accuracy of antigens is also affected by the labeling system. Due to the flexibility and size of the primary antibody and secondary Fab', the distance between the Au particle and the antigenic site can be as much as $15 \mathrm{~nm}$. Therefore, in spite of the resolution of HIM, the total localization accuracy is not better than the localization accuracy achieved using Au-labeling and conventional TEM or SEM, or fluorescence labeling and optical microscopy including photo-activated localization microscopy/stochastic optical reconstruction microscopy, structured illumination microscopy and stimulated emission depletion microscopy. The accuracy of immuno-SE-HIM can be improved by Au-conjugated Fab' labeling, which avoids the use of a secondary antibody. If Nanogold-Fab' labeling was combined with Au enhancement, the labeling density would probably be higher than the labeling density obtained with standard colloidal-Au-Fabs' employed for immuno-SE-HIM. The use of such labeling is expected to make high-resolution immuno-SE-HIM of deep specimen zones possible.

Cathode luminescence-based SEM and scanning TEM are widely used in material science (19). As demonstrated in the present study, IL-HIM can image ZnO-NPs above a dark background, and it has the potential to visualize signals behind nuclei and thick ER. Combined with multiple fluorescent labeling, IL-HIM promises multi-color-labeled microscopy at high-resolution, and -position accuracy, which would be useful to study the formation of molecular complexes. Fluorescent NP probes (20) directly bound to Fab' prepared from primary antibodies are required for this purpose.

In conclusion, the ability of HIM to realize high DOF observations for dried cells and Epon-embedded tissues, to detect Au tags employed in immunohistochemistry at high resolution and to exploit the IL signal make this microscopy an important and versatile novel tool for cell/tissue studies. A wide variety of Epon-based sample preparation methods developed in biological and medical science fields can now be tested. High-dose milling and mild-dose imaging by HIM could possibly be used in combination with EM for various 3D structure analyses. In addition, in the future, HIM of unstained and uncoated samples is expected to be applied in the fields of soft materials.

\section{Acknowledgements}

The authors would like to thank Dr T. Ebihara at the National Institute of Advanced Industrial Science and Technology (AIST) for valuable discussions. The authors would also like 

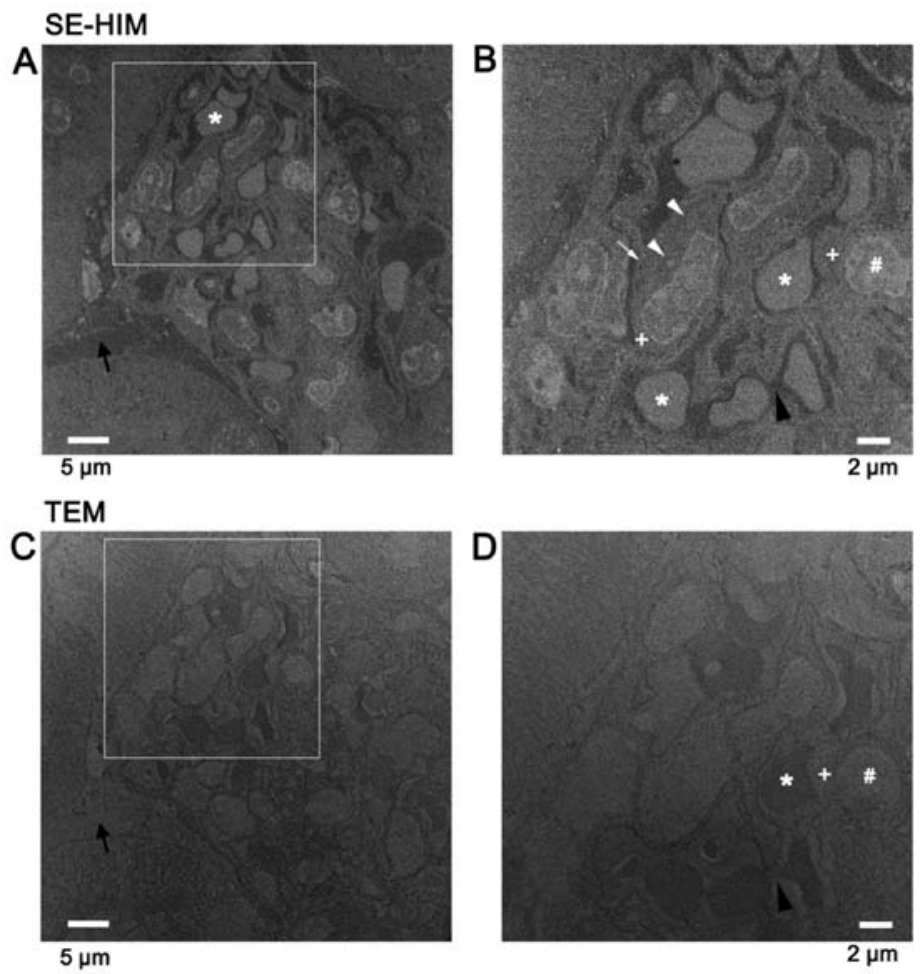

Figure 12. SE-HIM of Epon-embedded tissue sections. Unstained, uncoated Epon-embedded sections of mouse kidney tissue were observed on silicon nitride/silicon substrate by SE-HIM. (A) Glomerulus surrounded by the Bowman's capsule and convoluted tubules. The Bowman's space (black arrow) is dark. (B) Image of the rectangle in A. The Epon-filled extracellular gaps are dark. In glomerular capillaries, dark blood plasma (black arrowhead) surrounds homogeneously bright red blood cells $(*)$. Outside the capillaries, cells have a nucleus (\#) with peripheral bright densities (chromatin) and round blobs (nucleoli). Brightly contrasted blob-like and web-like structures (white arrowheads; possibly mitochondria and ER) and dark vesicle-like structures (white arrow; possibly vacuoles) can be distinguished in the cytoplasm (+). (C) A section close to the section imaged by SE-HIM in A was mounted on a copper 400 mesh grid and observed by TEM. In the TEM image, the Bowman's space (black arrow) is bright. (D) Image of the rectangle in C. Nuclei (\#) and cell organelles in the cytoplasm (+) can be distinguished. The extracellular gap is bright. Red blood cells ${ }^{*}$ ) in glomerular capillaries are homogeneously dark and surrounded by bright blood plasma (black arrowhead). The contrast in SE-HIM images was sometimes the reverse of the contrast in TEM images. SE-HIM, secondary electron-helium ion microscopy; TEM, transmission electron microscopy.
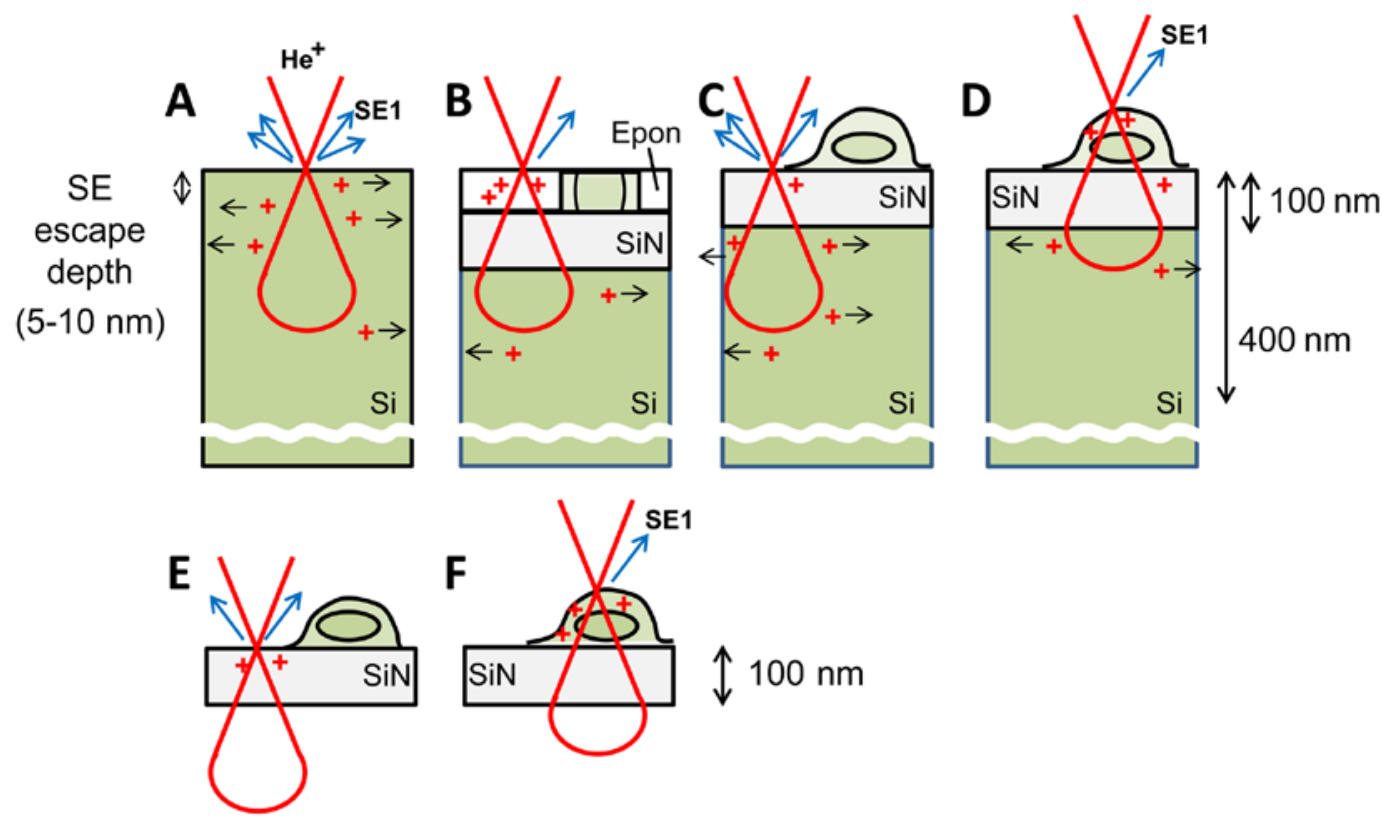

Figure 13. Model of SE-HIM imaging. (A) Si alone. Positive charges created by $\mathrm{He}^{+}$ions are mostly conducted to the surrounding Si. Residual charge near the surface partially precludes SEs from emitting from the 5-10 nm-thick surface zone. (B) Epon-embedded section on a SiN/Si bilayer. Since both Epon and SiN are insulators, positive charges hardly move to the Si layer, and stay near the surface. Conductive cell components (green) alleviate charging. (C and D) Cells dried on $\mathrm{SiN} / \mathrm{Si}$. (C) $\mathrm{SiN} / \mathrm{Si}$ is irradiated; charges are mainly created in the $\mathrm{Si}$ and minor charges in thin $\mathrm{SiN}$ tend to conduct to the underlying $\mathrm{Si}$ layer. (D) Cell on $\mathrm{SiN} / \mathrm{Si}$ is irradiated; positive charges stay near the cell surface, especially where intracellular membranous structure acts as an insulator to the Si layer. (E and F) Cells on SiN film. (E) SiN film is irradiated. Most $\mathrm{He}^{+}$ions penetrate the film, and positive charges remain there because the film is not lined by conducting Si. (F) Cell on SiN film is irradiated. Positive charges stay near the cell surface. SE-HIM, secondary electron-helium ion microscopy; Si, silicon; SiN, silicon nitride. 
to thank Mr. T. Iijima (AIST) and Dr Y. Morita (AIST) for the operation of the HIM at SCR station of AIST.

\section{Funding}

The present study was supported by a Grant-in-Aid for Structural Cell Biology (to CS), by grants from the Ministry of Education, Culture, Sports, Science, and Technology (MEXT) (to CS), by CREST (to CS), by a Grant-in-Aid for Scientific Research on Innovative Areas 'Sparse modeling' to CS, by a Grant-in-Aid for Scientific Research to CS from JSPS (15K14499), by a Grant-in-Aid from CANON to CS and by a Strategic Research grant from AIST to NISP (to CS).

\section{Availability of data and materials}

The images and datasets used and/or analyzed during the current study are available from the corresponding author on reasonable request.

\section{Authors' contributions}

CS and SO planned the project, designed the experiments, and performed the experiments together with MS, who also analyzed the data. CS wrote the paper with input from all co-authors. All authors read and approved the final manuscript.

\section{Ethics approval and consent to participate}

All experiments were approved by the Animal Care and Use Committee of the National Institute of Advanced Industrial Science and Technology (AIST; Tsukuba, Japan).

\section{Consent for publication}

Not applicable.

\section{Competing interests}

The authors declare that they have no competing interests.

\section{References}

1. Ananth M, Scipioni L and Notte J: The helium ion microscope: The next stage in nanoscale imaging. Am Lab 40: 42-46, 2008.

2. Boden SA, Franklin TMW, Scipioni L, Bagnall DM and Rutt HN: Ionoluminescence in the helium ion microscope. Microsc Microanal 18: 1253-1262, 2012.

3. Bell DC: Contrast mechanisms and image formation in helium ion microscopy. Microsc Microanal 15: 147-153, 2009.
4. Cohen-Tanugi D and Yao N: Superior imaging resolution in scanning helium-ion microscopy: A look at beam-sample interactions. J Appl Phys 104: 063501-063507, 2008.

5. Inai K, Ohya K and Ishitani T: Simulation study on image contrast and spatial resolution in helium ion microscope. J Electron Microsc (Tokyo) 56: 163-169, 2007.

6. Postek MT, Vladar A, Archie C and Ming B: Review of current progress in nanometrology with the helium ion microscope. Meas Sci Technol 22: 024001-024014, 2011.

7. Morgan J, Notte J, Hill R and Ward B: An introduction to the helium ion microscope. Micros Today 14: 24-31, 2006.

8. Notte J, Hill R, McVey S, Farkas L, Percival R and Ward B: An introduction to the helium ion microscope. Microsc Microanal 12: 126-127, 2006.

9. Rice WL, Van Hoek AN, Păunescu TG, Huynh C, Goetze B, Singh B, Scipioni L, Stern LA and Brown D: High resolution helium ion scanning microscopy of the rat kidney. PLoS One 8: e57051, 2013.

10. Bazou D, Behan G, Reid C, Boland JJ and Zhang HZ: Imaging of human colon cancer cells using He-Ion scanning microscopy. J Microsc 242: 290-294, 2011.

11. Chen X, Udalagama CNB, Chen C-B, Bettiol AA, Pickard DS, Venkatesan $\mathrm{T}$ and Watt $\mathrm{F}$ : Whole-cell imaging at nanometer resolutions using fast and slow focused helium ions. Biophys J 101: 1788-1793, 2011.

12. Maruyama Y, Ebihara T, Nishiyama H, Suga M and Sato C: Immuno EM-OM correlative microscopy in solution by atmospheric scanning electron microscopy (ASEM). J Struct Biol 180: 259-270, 2012.

13. Memtily N, Okada T, Ebihara T, Sato M, Kurabayashi A, Furihata M, Suga M, Nishiyama H, Mio K and Sato C: Observation of tissues in open aqueous solution by atmospheric scanning electron microscopy: Applicability to intraoperative cancer diagnosis. Int J Oncol 46: 1872-1882, 2015.

14. Nishiyama H, Suga M, Ogura T, Maruyama Y, Koizumi M, Mio K, Kitamura S and Sato C: Atmospheric scanning electron microscope observes cells and tissues in open medium through silicon nitride film. J Struct Biol 169: 438-449, 2010.

15. Maunsbach AB and Afzelius BA: Immunocytochemistry. In: Biomedical Electron Microscopy. Academic Press, San Diego, pp383-426, 1999.

16. Ward BW, Notte JA and Economou NP: Helium ion microscope: A new tool for nanoscale microscopy and metrology. J Vac Sci Technol B 24: 2871-2874, 2006.

17. Kataoka-Hamai $\mathrm{C}$ and Miyahara Y: Field-effect detection using phospholipid membranes. Sci Technol Adv Mater 11: 033001-033005, 2010.

18. Ura K: Electron beam interaction with specimen. In: Electron Beam Testing Technology. Thong JT (ed). Springer, pp175-209, 1993.

19. de Abajo FJG: Optical excitations in electron microscopy. Rev Mod Phys 82: 209-275, 2010.

20. Glenn DR, Zhang H, Kasthuri N, Schalek R, Lo PK, Trifonov AS Park H, Lichtman JW and Walsworth RL: Correlative light and electron microscopy using cathodoluminescence from nanoparticles with distinguishable colours. Sci Rep 2: 865, 2012.

This work is licensed under a Creative Commons Attribution-NonCommercial-NoDerivatives 4.0 International (CC BY-NC-ND 4.0) License. 